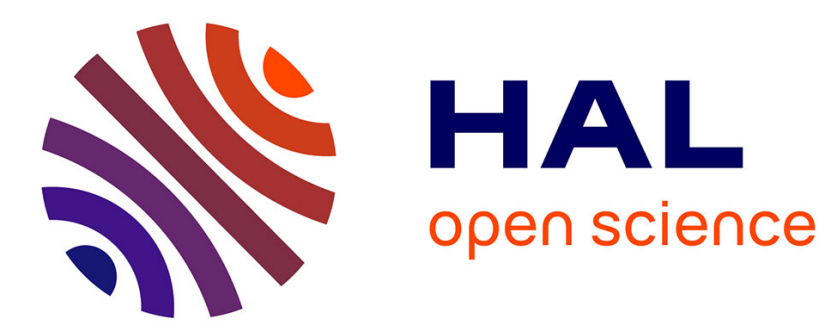

\title{
Contraintes de site à Rotomagus
}

Jean-Louis Ballais

\section{To cite this version:}

Jean-Louis Ballais. Contraintes de site à Rotomagus. Bulletin de Rouen Archéologie, 1983, 3, pp.1725. hal-01544919

\section{HAL Id: hal-01544919 https://hal-amu.archives-ouvertes.fr/hal-01544919}

Submitted on 22 Jun 2017

HAL is a multi-disciplinary open access archive for the deposit and dissemination of scientific research documents, whether they are published or not. The documents may come from teaching and research institutions in France or abroad, or from public or private research centers.
L'archive ouverte pluridisciplinaire HAL, est destinée au dépôt et à la diffusion de documents scientifiques de niveau recherche, publiés ou non, émanant des établissements d'enseignement et de recherche français ou étrangers, des laboratoires publics ou privés. 


\section{ROUEN}

\section{ARCHÉOLOGIE}

AGGLOMERATION ROUENNAISE

HOTEL DES SOCIÉTES SAVANTES

190, rue Beauvoisine, 76000 ROUEN

C.C.P. ROUEN $2800.96 \mathrm{~F}$

\section{bulletin}

MAI 1983

SOMMAIRE:

Compte-rendu de l'Assemblée Générale du 21/10/82

Statuts de l'Association.

Libre Propos: sur une exposition (J. Vavasseur).

Fouille de la Rue aux Juifs à Rouen (P. Halbout).

Contraintes de site à Rotomagus (J.L. Balais).

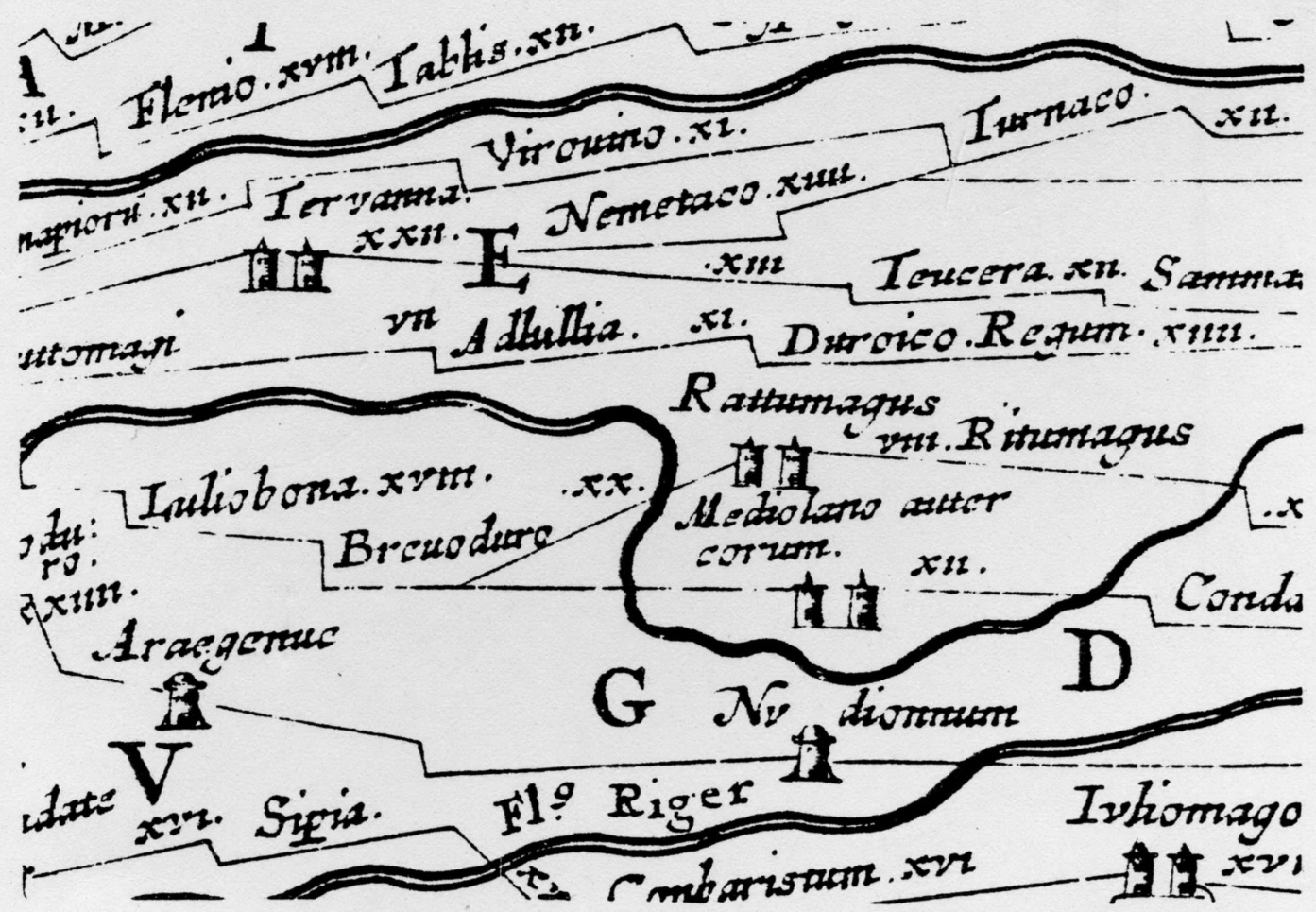

Extrait de la Table de Peutinger. 


\title{
CONTRAINTES DE SITE A ROTOMAGUS
}

\begin{abstract}
$* * * * * * * * * * * * * * * * * * *$
L'essor vigoureux de la recherche archéologique urbaine à Rouen depuis quelques années, dans des conditions difficiles (Rouen GalloRomain,1982) a rapidement posé la question des limites de la ville romaine et de leurs variations au cours du temps. Sans nier le rôle des facteurs humains, sociaux ou économiques, il est apparu assez vite que les con-traintes naturelles du site avait dû peser lourd. Profitant de l'exeption-nelle richesse de la ville de Rouen en documents cartographiques à grande échelle et en sondages ( cf. bibliographie), j'ai tenté de préciser certaines de ces contraintes.
\end{abstract}

\section{I-UN SITE TRES PARTICULIER:}

\section{UNE TERRASSE DE RIVE CONCAVE DE M!EANDRE}

Rouen occupe un site à priori banal: un méandre de fleuve, comme Paris ou Londres. Cependant, la comparaison avec Paris, ou plutôt Lutèce, tourne vite court. En effet, née dans l'île de la Cité, Lutèce s'est ensuite développée sur la rive convexe de méandre qui se relève doucement vers le sud. Au contraire, Rotomagus s'installe puis se développe sur la rive concave de méandre. Trés généralement, ce type de rive peut présenter deux topographies opposées, mais tout aussi dé-favorables à l'installation d'une ville. Ou bien, par suite de la dynamique même du méandre, la rivière coule juste au bord du plateau (comme à l'ouest de Grand Couronne ou à Caudebec). Ou, au contraire, le méandre ayant migré, la rivière s'est écartée du plateau dont elle est séparée dorénavant par des terrains bas, meubles et marécageux (comme sur la rive droite à Paris).

L'originalité de Rouen provient de ce qu'une terrasse s'est maintenue en rive concave. Des possibilités d'extension se présentaient pour la ville car, outre la terrasse, existent aussi des espaces plans et peu élevés au-dessus de la Seine, grâce à une zone de confluences mul-tiples, surtout avec le Robec (grossi de l'Aubette). Ces affluents présent-ent des vallées assez larges (500m à Darnétal) et à fond plat. Enfin, des vallons secs pouvaient faciliter les relations avec le plateau de Caux.

En somme, la localisation du site de Rouen peut s'envisager

à deux échelles différentes: celle du méandre et celle de la terrasse. 


\section{1- la localisation du méandre :}

L'étude de la formation et de l'évolution des méandres a l'ongtemps constitué un morceau de choix pour les géomorphologues, provoquant de vigoureuses controverses qui paraissent bien dépassées aujourd'hui. Le méandre de Rouen présente cependant un intérêt particulier dans la mesure ou, s'intégrant dans l'ensemble des méandres de la BasseSeine il doit donc son existence à la dynamique fluviale, mais ses carac-téristiques sont influencées par la structure géologique locale, en l'espèce un anticlinal faillé.

\section{a - l'anticlinal de Rouen}

Avec des pendages trés faibles (quelques degrés), il a une orientation générale NW-SE. Dans le détail, il est NNW-SSE au sud puis franchement NW-SE au nord-ouest ou il est recoupé par la Seine. La culmination se place au niveau de la Sablière, à l'WSW de l'ile Lacroix ou les dépôts récents discordants recouvrent les alternances de sables, de grès et de calcaires gréseux du Portlandien. Plus au Nord, un léger abaissement d'axe permet aux calcaires sublithographiques gris bleutés, entrecoupés de lits marno-argileux (sommet du Portlandien), de constituer le substratum alors que, sous la Seine, alternent des sables, des argiles et des grès. Dans le détail, l'anticlinal présente un flanc NE à pendage plus faible qu'au SW.

\section{b - le substratum de Rotomagus:}

Du fait de la disposition de l'anticlinal, le Portlandien ne constituait qu'une faible partie du substratum de Rotomagus, réduite à un mince liseré sur la rive droite de la Seine et qui ne dépasse pas, au nord, la rue du Général Leclerc. Il est à une altitude variant de $-7 \mathrm{~m}$ à $-11 \mathrm{~m}$ N.G.F.

L'essentiel du substratum de Rotomagus est constitué des différents faciès de l'Albien. Quoi que discordante, l'argile de base, glauconieuse, parfois sableuse, épaisse d'une dizaine de mètreş; constitue régulièrement le flanc NNE de l'anticlinal au sud de la cathédrale et atteint la moitié Sud de l'Hotel-Dieu, à l'ouest. Les sables verts quartzeux, fins à grossiers, à quelques intercalations argileuses, épais de $25 \mathrm{~m}$, forment une "excrois-sance" dans la vallée du Robec et s'étendent, au nord, jusqu'à SaintOuen et jusqu'au square Vedrel. Enfin, les argiles supérieures ou argiles du Gault, noires, compactes, à lits de sables micacés, épaisses de $23 \mathrm{~m}$, atteignent le sud du lycée Corneille, englobent le lycée Fontenelle et coupent en deux la place Saint Hilaire. L'altitude maximum de l'Albien varie de -13 à $+15 \mathrm{~m}$ N.G.F.

Les terrains plus récents: craies du Cénomanien, du Turonien, du Sénonien et du Coniacio-Santonien inférieur n'affleurent qu'à la base ou sur les versants du plateau septentrional. 


$$
c \text { - les failles }
$$

L'anticlinal est affecté de quelques failles, de faible rejet en général, en nombre variable selon les documents. La principale est la faille de la Seine, de même orientation que l'anticlinal. Elle est dis-continue. Vers le NW, elle coupe la presqu'ile Rollet en se dédoblant avec un abaissement des compartiments Nord et des rejets de quéTques mètres. Les quelques failles transversales ont des rejets comparables. L'une d'entre elles, orientée WNW-ESE vient affecter la côte Sainte Catherine ou elle a orienté le vallon d'Eauplet à l'est du tunnel de la voie ferrée (Carte lithologique au 1/5000)

\section{d- conséquences :}

Cette structure complexe, caractéristique de la bordure NW du bassin parisien, ne se traduit pas dans le relief, à la différence du pays de Brāy: les plateaux, de part et d'autre de la Seine, restent hori-zontaux, nivelés qu'ils ont été par une ou plusieurs surfaces d'aplanis-sement tertiaires. Par contre, on peut se demander si le pendage vers l'ENE, dans des calcaires, sur le flanc oriental de l'anticlinal, ne favorise pas le glissement de la Seine dans cette direction, accentuant la dynamique propre du méandre. Enfin, le seuil constitué par l'anticlinal a pu faciliter la traversée de la Seine au niveau de Rotomagus, dans la mesure ou il provoque un ralentissement du courant immédiatement à l'amont.

\section{$\underline{2}$ - LA TERRASSE DE ROUEN}

Si l'on fait abstraction de l'épaisseur considérable des remblais (6 à $8 \mathrm{~m}$ en moyenne). La terrasse a une forme ovoïde dont la limite nord passe légèrement au sud et parallèlement à la rue Thiers. La pointe occidentale arrive un peu au SW de la place Cauchoise. De là, vers le SE, la limite rejoint les remblais de bord de seine en face du pont Jeanne d'Arc, puis le carrefour rue du général Leclerc / rue de la République, passe à l'ouest du carrefour de la rue Ruissel et de la rue Eau de Robec, sous l'église saint Ouen et rejoint le sud de la rue Thiers. L'altitude de son sommet varie de $-1 \mathrm{~m}$ NGF à la Haute Vieille Tour à $+4 \mathrm{~m}$ à la Cathédrale et $+8 \mathrm{~m}$ au nord de la rue du Gros Horloge.

Curieusement, sa composition pétrographique reste trés mal connue, en raison d'une urbanisation trés ancienne, d'une part, et, d'autre part, de la reconnaissance tardive de l'originalité de cette terrasse ( $M$. Porcher, 1977). C'est ainsi que les cartes des formations superficielles au $1 / 25000$ et au $1 / 10000$ la représentent comme constituée d'alluvions des rivieres affluentes et non d'alluvions de la Seine. Par contre, D. Alduc et al. (1979) l'intègrent dans la série des terrasses du fleuve. En fait, la terrasse de Rouen présente une discontinuité au niveau de la rue du Gros Horloge. Au nord, sous $1,50 \mathrm{~m}$ de limons, $3,5 \mathrm{~m}$ de grave reposent sur les sables verts albiens et se teminent en biseau vers le sud ou existe une épaisseur comparable de grave se terminant en biseau également, mais vers le nord 
L'étude ponctuelle mais détaillée de la jonction de ces deux nappes alluviales, place de la Cathédrale, a mis en évidence l'absence de liaison entre elles (J-P. Leriverend, in littéris). Les études granulomètriques faites par la C.E.T.E. de Rouen montrent que la nappe alluviaTe sup-érieure est trop pauvre en sables pour faire partie du système séquanien. En réalité, la terrasse de Rouen pourrait donc se décomposer en deux parties: une nappe alluviale déposée pour l'essentiel par le Robec et ses affluents, au nord de la rue du Gros Horloge, et une nappe alluviale déposée par la Seine au sud ( M. Voiment, comm. orale). Dés l'ouverture d'un prochain chantier important, le prélèvement d'échantillons pourra permettre de préciser cette pétrographie et donc l'origine des nappes alluviales.

\section{a - la terrasse de Rouen est-elle déformée?}

La nappe alluviale de Rouen vient d'être attribuée récemment (D. Alduc et al. 1979)/Elle constitue une portion d'un niveau de terrasse que l'on suit de Léry, à l'amont, au Havre, à l'aval. Bien que ce niveau paraisse bien établi actuellement, on peut poser la question de la dé-formation éventuelle de la nappe de Rouen, d'autant que le profil longitudinal du niveau d'ablation qui supporte les cailloutis $c a$ fond (plus recents que la nappe de Rouen) montre, en aval de Caudebec un palier peut-être dû au rejet de la faille de Bolbec-Fécamp ou de celle de Villequier (D. Alduc et al._1979).

En effet selon une hypotèse de J.P. Leriverend (comm. orale), des failles guideraient le tracé des vallons du plateau septentrional et, en particulier, celui des deux principaux. Le vallon occidental est en réalité le résultat de la confluence de trois élements. Le plus long descend du hameau de la Vielle et conflue dans le quartier saint André (cote 46) avec un affluent occidental descendu du hameau des Cottes. Un affluent oriental descend du mont Fortin. L'ensemble aboutit, en gros, dans l'axe des rues Vertes puis Jeanne d'arc. Le second vallon principal commence entre Bois-Guillaume et Bihorel (son versant NNW est occupé par la cité Jeanne d'Arc), est emprunté par la rue Bonnefol et aboutit immédiatement à l'est de Saint Ouen. Des travaux récents (C.E.T.E. 1979) ont confirmé l'existence, à peu prés dans l'axe de la rue Verte, d'une faille de 10 à $15 \mathrm{~m}$ de rejet.

Ces vallons sont d'ailleurs plus anciens que la nappe de Rouen car ils entaillent nettement le substratum (carte lithologique du substratum au 1/5000). Le vallon occidental étant trés proche de la bordure occidentale de la nappe de Rouen et le vallon oriental coïncidant avec la limite Est, on pouvait envisager un rejet des failles guidant ces vallons, dans le sens d'un léger soulèvement en horst entre elle deux. Ce rejet daterait donc, au plus tard, du Pleistocène supérieur, ce qui paraît bien récent pour une région longtemps̄ réputée tectoniquement stable. Cependant, il est maintenant acquis que l'ex-trémitée occidentale du Pays de Caux s'est relevée de plusieurs dizaines de mètres vers la fin du Pléistocène inférieur ou au début du Pléistocéne moyen (D. Alduc et al. 1979) De même, la faille de Rouen a rejoué au cours de l'Holocéne entré le Houlme et Pavilly (R. Wyns, 1977). 
Cependant, l'étude des tremblements de terre à Rouen montre que la stabilité n'est pas atteinte. En effet des tremblements de terre auraient été enregistrés en $890,1136,1142,1315,1522$, $1580,1691,1692,1711,1755,1756,1769,1773,1775,1797,1798$, 1799,1803 (?), 1829, 1866, 1878, 1885, 1889, 1927, ( L. Lecornu (18881889), P. Lemoine (1911), J. Vogt (1979). Cette longue liste doit être appréciée en fonction des réserves de méthode émises par J. Vogt (1979, p9-15) concernant la reconnaissance des tremblements de terre. En particulier, P. Lemoine (1911) s'appuie sur des travaux de Perrey dont "on ne peut s'inspirer sans esprit critique". Seuls les tremblements de terre du 6-4-1580 (intensité VI-VII à Rouen : verriéres, des églises et des maisons brisées, quelques parties des voûtes de la cathédrale s'écroulent ), de 1756 et de 1866 ont eu un caractère général dans le Bassin Parisien ( $P$. Lemoine, 1911). Les seuls bien connus par leurs rapports avec la structure géologique sont ceux du ler/12/1769 (inten-sité V à Rouen) et de 1845 (non ressenti à Rouen). Le séisme de 1769 a été signalé dans plusieurs localités qui paraissent s'échelonner sur la faille de la Seine, surtout à ses deux extrèmités: Rouen et Paris. Le séisme de 1845 à été ressenti entre Yvetot, Bacqueville et Maromme, soit de part et d'autre du prolongement de la faille de Rouen. Dans un Bassin Parisien trés peu sismique, Rouen est le seul point ou un séisme de magnitude égale ou supérieure à 3 ait été enregistré par les instruments entre 1962 et 1976 pour tout le quart Nord-ouest (J. Vogt, 1979, fig. 8).

On peut donc en conclure que la faille de la Seine est peut-être encore active, mais rien $n$ 'indique que se soit le cas de celles qui lui sont globalement transversales. Il était donc nécessaire d'essayer de mettre en évidence un rejeu de cette faille. L'étude des nombreux sondages effectués pour le port Autonome de Rouen dans la darse Charles Babin ainsi qu'au nord et au sud, de part et d'autre de la faille de la Seine dédoublée, permet difficilement de conclure. Globalement, les épaisseurs de la grave weichselienne sont plus importantes sur les sables verts et argiles de Gault (8 à 17m) que sur la craie $(4$ à $10 \mathrm{~m})$ Ceci peut très bien s'interpréter comme le résultat de l'érosion différentielle puis de l'accumulation, ce que font les coupes géologiques de la carte lithologique du substratum au 1/10000. J'ai donc entrepris une étude plus fine, de part et d'autre des failles.

A l'ouest de la faille occidentale supposée, sur le compar-timent ręlevé, les épaisseurs de grave sont de l'ordre de 4 à $9 \mathrm{~m}$ A l'est, sur le compartiment abaissé, elles sont nettement plus im-portantes: 8 à $10 \mathrm{~m}$. Le compartiment occidental ayant un substratum crayeux et le compartiment oriental un substratum sableux, l'érosion différentielle peut encore être invoquée, mais on peut penser aussi à un fonctionnement de la faille pendant le dépôt qui se serait donc effectué de manière préférentielle sur le compartiment oriental affaissé Cependant, les sondages 56 et $56 \mathrm{~A}$, trés proches mais de part et d'autre de la faille supposée, infirment cette dernière hypothèse: en effet, ils ont traversé une épaisseur presque identique de grave respectivement $8,65 \mathrm{~m}$ et $8,25 \mathrm{~m}$, alors que le substratum de $56 \mathrm{~A}$ est constitué de sables albiens.

A l'ouest de la faille orientale, sur le compartiment sou-levé, les épaisseurs de grave varient de 12 à $19 \mathrm{~m}$ et, sur le com-partiment oriental, de 8 à $17 \mathrm{~m}$, ce qui n'est pas significatif. D'autant plus que les sondages 38 et 36 , de part et d'autre de la faille montrent des épaisseurs de grave identiques $(17,95 \mathrm{~m}$ et $17,20 \mathrm{~m})$ sur un sub-stratum identique de sable vert albiens. 
le site initial de Rotomagus :

Jusqu'au ler siècle aprés J.C. , Rotomagus est situé dans une zone comprise entre les rues de l'Hopital et Ganterie au Nord, la rue de la République à l'Est, la rue du Gros Horloge et la rue Sainte Croix des Pelletiers au Sud et à l'Ouest (Rouen gallo-romain 1982, p4) Acette époque donc, la ville est fondée strictement sur la nappe alluviale de Rouen, parfois par l'intermédiaire de limons qui s'avancent au sud jusqu'à proximité de la cathédrale actuelle. Si la ville n'atteint pas les limites de la nappe, au sud et à l'ouest, elle. s'en rapproche au nord et l'atteint déjà pratiquement à l'est. Cependant, au sud, elle vient probablement au contact de la tourbe qui repose sur la nappe de grave (coupe 1-1', carte lithologique du substratum au $1 / 5000$ )

A ce momment Rotomagus est une ville de terrasse, de vallée, pas encore un port.

\section{2- La première extention, au ler siècle :}

Elle se fait surtout vers le sud ou apparaît le premier port (Rouen gallo-romain, 1982, p 4). Cette extention se développe encore, pour l'essentiel, sur la nappe alluviale, mais aussi en partie sur les limons argileux et sables coquillers holocènes qui ravinent la grave en bordure de la Seine (coupe 1-1', carte lithologique du substratum au $1 / 5000$ )

Elle suppose déjà une maitrise importante de l'hydrologie locale. En effet, au niveau de la discontinuité de la terrasse de Rouen, la nappe phréatique perchée dans la grave supérieure (qui draîne les eaux qui descendent du nord) est en charge (environ $50 \mathrm{~cm}$ ). L'évacuation de cette nappe a imposé le creusement d'égoûts volumineux, mais aussi l'accumulation de remblais, en particulier prés du port, à la Haute Vieille Tour, oy ils peuvent s'interpréter non seulement comme liés au port, mais aussi comme permettant de placer hors eau une plateforme arrière. D'autre part, l'abondance des sources, au contact de la craie et de l'argile de Gault, captées par des tuyaux de bois a permis une distribution particulière trés développée, ce qui n'est pas le cas habituellement dans une ville Gallo-romaine (P. Halbout, in litteris) . Cette circulation particulière explique l'absence de château d'eau et d'aqueduc, mais aussi le puits artésien de la Haute Vieille Tour (idem).

\section{3- La ville de la fin du Ilème siècle :}

L'extension vers le SW permet d'atteindre la limite de la nappe de Rouen alors que, vers l'ouest, on en reste encore assez loin. Mais c'est vers le nord que la limite est largement dépassée, aussi bien par la construction de l'amphithéatre que par celle de quartiers artisanaux (Rouen gallo-romain, 1982, p 7) . 
Là, en effet, la nappe alluviale de rouen se termine ou disparait sous les formations solifluées descendues des versants du plateau de caux qui s'approchent trés prés de la rue Thiers. Vers le Nord, leur épaisseur atteint rapidement plusieurs metres. Elles sont constituée d'une matrice de limons éoliens remaniés emballant des blocs de craie comme place Beauvoisine ou au nord de l'Hotel de Ville.

Cependant, ces blocs n'atteingnent ni le nombre, ni le volume de ceux qui recouvrent le versant occidental du mont Gargan, face à l'ile Lacroix (carte lithologique du substratum au 1/10000). Ces for-mations solifluidales recouvrent non seulement la grave weichselienne, mais aussi les alluvions fines holocénes (idem). Encore aujourd'hui, tout le quartier au nord de la rue Thiers est fondé sur des blocs de craie soliflués (J-P. Leriverend, comm. orale).

Seules, certaines nécropoles (route de Lillebonne, rue Saint-Hilaire) sont installées dans les alluvions limono-argileuses et tourbeuses, à l'ouest ou à l'est de la nappe de Rouen.

\section{4 - La ville du 4ème siècle:}

Aprés un déclin assez lent, Rotomagus s'entoure tardivement d'une enceinte presque carrée d'un peu moins de $500 \mathrm{~m}$ de coté (Rouen gallo-romain, R $11-12$ ). A nouveau, comme aprés l'extention du ler siècle, la ville se réduit à la majeure partie de la nappe alluviale.

\section{5 - Une limite presque constante à l'Est :}

Tout au cours de son histoire, Rotomagus n'a pratiquement pas dépassé, à l'est, l'emplacement actuel de Saint Ouen, alors qu'aucun obstacle n'apparait. C'est du coté de l'hydrologie qu'il faut chercher. En effet, nous sommes là à l'extrémité méridionale du vallon de la rue Bonnefoi. Commes les autres, il est colmaté par du loess colluvionné. S'il ne possède plus d'écoulement superficiel, par contre, il est encore l'objet de drainage en profondeur ( J-P. Leriverend, comm. orale) et constituait un obstacle redoutable à l'urbanisation par ses dépôts meubles et humides. Le même type d'ob-stacle était constitué, à l'ouest, par le vallon de la rue verte mais, moins entaillé dans le substratum et ne bordant pas une vallée impor-tante comme celle du Robec, il a moins longtemps limité l'urba-nisation. Enfin, l'importance du vallon de la rue Bonnefoi a été re-connue plus tard, quand on l'a utilisé pour détourner le Robec qui coulait au ras de la côte Sainte Catherine (C.E.T.E., 1980)

\section{CONCLUSION :}

La richesse exeptionelle de la documentation sur la géologie de Rouen permet donc d'éclairer quelques point concernant Rotomagus et son site, à bien des égards aussi exeptionnels. 
On peut en retenir l'idée que la ville romaine, sa fondation et son extention, sont restées liées à la nappe alluviale de Rouen, sur la rive concave d'un méandre de la Seine. A partir du site primitif sur la partie haute de cette terrasse weichselienne, les habitants de Roto-magus se sont heurtés surtout à des problèmes hydrologiques lors de l'extension de leur ville. Ils en ont résolu la plupart, ont été arrêtés par certains. Ce n'est que lors de la phase maximale d'extention que la ville a débordé sur les dépôts solifluidaux au nord, voire sur les alluvions fines holocènes à l'ouest et à l'est. Mais c'est sur la terrasse graveleuse qu'elle s'est repliée au IVème siècle.

Cependant, plusieurs problèmes ne sont pas encore résolus. En particulier, l'étude a été conduite en admettant implicitement que la Seine occupait le même tracé qu'aujourd'hui, ce qui est sujet à caution et peut avoir eu une influence sur l'extension de la ville, en particulier à l'ouest du port du ler siècle. Ou encore, et pour se limiter aux problèmes archéologiques, si Rotomagus supplante Juliobona (Lillebonne) au Ilème s., quelle est la part des facteurs naturels, en particulier de la transgression flandrienne (Rouen galloromain, 1982, p6) ? Grâce à l'abondance des documents, des cartes et des sondages en particulier, on peut espérer prochathement apporter des réponses a ces questions.

REMERCIEMENTS: Outre J-P. Leriverend, ingénieur en chef, directeur de la voie publique à Rouen, sans qui cette étude serait restée bien théorique, je remercie vivement MM. Calais, ingénieur au port autonome et Bérard, ingénieur I.A.M. , chef des bureaux d'études au port auto-nome de Rouen qui nous ont largement ouvert l'accès aux résultats des forages, M. Voiment, directeur du service géologique du C.E.T.E., Patrick Halbout, qui $\mathrm{m}$ 'a demandé ce travail qui lui doit beaucoup, le centre de recherches archéologiques de Haute Normandie et toute l'équipe de "ROUEN-ARCHEOLOGIE" .

\section{Documents consultés}

C.E.T.E. (s.d.) : Carte lithologique (écorché) du substratum au 1/5000 Div. Lab. , section Géol.

ORGANISME TECHNIQUE REGIONAL DES PONTS ET CHAUSSEES (1969) : carte lithologique (écorché) du substratum de l'agglomération rouennaise au $1 / 10000$

(1969) : carte géomorphologique de l'agglomération rouennaise au $1 / 25000$

(1969) : carte des formations superficielles de l'agglomération rouen-naise au $1 / 25000$

(1969) : idem au 1/10 000

(1969) : carte de l'aptitude des sols aux fondations, agglomération rouennaise au $1 / 25000$

(1969) : etude géotechnique du site de l'agglomération rouennaise 5 vol., coupes

(1979) : carte répertoire des sondages, collecteur de ceinture nord tunnel, profil en long géotechnique, au 1/100 et 1/1000

(1980) : idem voie est de Rouen entre le pont Mathilde et la rocade nord , etude géotechnique

(1911-1969) : sondages divers 
Alduc D. , Auffet I-P, Carpentier G. , Lautridou J-P, LefebvreD. et Porchet $M$.

(1979) : Nouvelles données sur le Pléistocène de la basse vallée de la Seine et son prolongement sous-marin en Manche orientale

Bull. d'inform. des géol. du bassin de paris, vol. 16, n², p 27/34, 4fig., 1 tabl.

Hommeril P. (1977) : le site géologique de l'agglomération rouennaise, in "quides géologiques regionaux : Normandie ", par F. Doré, P. Juignet, C. Larsonneur, C. Pareyn et M. Rioult, Masson, p188/189.

Lecormu L. (1888/1889) : les tremblements de terre en Normandie. Bull. Soc. Linn. de Normandie, p235/253

Porcher M. (1977) : Lithostratigraphie des alluvions fluviatiles holocènes de la basse vallée de la Seine. Bull. Soc. Géol. Nomandie, tome LXIV, fasc. 4 , p181/201

Rouen gallo romain (1982): Catalogue de l'exposition du musée des Beaux Arts de Rouen_XIV + 116p. plan fig. et phot.

Vogt J. (1979) : les tremblements de terre en France Mémoires du BRGM n०96,220p

Wyns R. (1977) : tectonique récente dans l'ouest du bassin de Paris : méthodes d'étude et bilan des déformations plio-quaternaire Bull. Soc. Géol. Fr., (7), $t$ XIX, p 1093/1101, 3 fig. 